\title{
A Stereocontrolled Synthesis of D-erythro-Sphingosine and D-ribo-Phytosphingsine ${ }^{\dagger}$
}

\author{
Sung Ho Kang, ${ }^{*}$ Yu Sang Hwang, and Hee Seung Lee \\ C'enter for Molecular Design and Sinthesis, Deparment of (hemistry, School of Molecular Science (BK2I), \\ Korea thanced Institute of Sicience and Technologw, Daejeon 305-701, Korea \\ Received.April 1, 2002
}

Key Words : $\beta$-Amino alcohols, D-erythro-Sphingosine, D-riho-Sphingosine

Since a variety of physiologically valuable compounds comprise $\beta$-anino hỵdroxy ethylene subunits. 'we have been engaged in developing stereoselective synthetic routes to $s y$ - and $a m i-\beta$-amino alcohols. The routes have been established by the electrophile-promoted intramolecular anidations of ally lic ${ }^{2}$ and homoally lic trichloroacetimidates. ${ }^{3}$ in which the stereochemistry is conceivably controlled by either steric or electronic effects. Splingosine derivatives. regarded as $\beta$ amino alcohols, have attracted considerable attention due to their cnucial roles ${ }^{4}$ in a number of biological functions including inhibitory activity against protein kinase $\mathrm{C} .{ }^{5}$ Ther are essential components of sphingolipids. e.g., cerebrosides. gangliosides, sphingomyelins and ceramides. Sphingolipids and their metabolites are involved in signal transduction, cell regulation. and cell recognition such as growth. differentiation. adhesion and the immune response. ${ }^{7}$ In addition, many glycosphingolipids from marine organisms display pronounced antitumor. ${ }^{\text {" }}$ antiviral. ${ }^{9}$ antifungal. ${ }^{11}$ antiinflammator. ${ }^{11}$ inmunosuppressive. ${ }^{12}$ immunostimulatory, ${ }^{13}$ neuritogenic ${ }^{14}$ and cytotoxic activities. ${ }^{15}$ The biochemical and biomedical significance of sphingosine-containing compounds as well as the synthetic utility of our developed methodology for anti- $\beta$-amino alcohols ${ }^{30}$ led us to choose (-)-D-enthrosphingosine 1 and (+)-D-ribo-phy tosphingosine 2 as the synthetic targets. ${ }^{16}$ In this paper we describe a convenuent stereoselective synthesis of the two sphingosines 1 and 2 starting from dilydro-1,3-oxazines 4 and 10 . respectively.

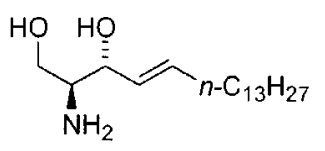

1

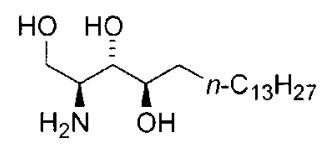

2
The synthesis of D-enthro-sphingosine 1 began with dihydro-1,3-oxazine 4. which was prepared in 5 steps and $68 \%$ overall yield from triol 3 . Alternatively 4 could be yielded more efficiently as described in the following (Scheme 1). After disily lation of 3 , the generated disily l ether was treated with $\mathrm{Cl}_{3} \mathrm{CCN}$ in the presence of $\mathrm{NaH}$ and $n-\mathrm{Bu}_{4} \mathrm{NF}$ to effect chemoselective monodesilylation and monoinidate formation. The resulting silyloxy homoally lic imidate was iodoamidated using $\mathrm{IBr}$ to give the desired stereoisomeric dilhydro-1.3-

'This paper is dedicated to the late Professor Sang Chul Shim at KAIST. oxazine $+\left([\alpha]_{1)}^{26}-21.5\right.$, c $\left.1.0 . \mathrm{CHCl}_{3}\right)$ exclusively in $85 \%$ overall yield. The iodohydrin functionality of 4 was reductively eliminated by sequential addition of trifluoroacetic anhydride and $\mathrm{NaI}$ to fumish alkene. The alkene was completely hydrolyzed and then protected to provide dihydrosy carbanate $5\left([\alpha]_{1,}^{26}-5.9 .<1.1 . \mathrm{MeOH}\right)$ in $92 \%$ overall yield from 4 . The olefinic double bond of 5 was ozonized and reduced. The resultant triol was converted into 6-membered benzylidene $6\left(\mathrm{mp} .152-153^{\circ} \mathrm{C}:[\alpha]_{1)}^{20}+2+.5 .61 .0 . \mathrm{MeOH}\right)$ in $65 \%$ overall yield from 5 . Swern oxidation of $6^{17}$ and the subsequent modified Julia olefination ${ }^{1 *}$ with 7 afforded a 2.8 : 1 mixture of trans- and cis -alkenes. $8 \mathrm{t}$ and $8 \mathrm{c}$. in $71 \%$ combined yield. After chromatographic separation. 8t $\left([\alpha]_{1)}^{20}\right.$ $\left.+16.9 . c^{\prime} 1.5 . \mathrm{CHCl}_{3}\right)$ was hydrolyzed to produce $\mathrm{D}-\mathrm{e} \eta \mathrm{y} / \mathrm{hro}$ -<smiles>CC(C)(O)/C=C/[C@H](O)CO</smiles><smiles>CC(C)=C[C@@H](NC(=O)OCc1ccccc1)[C@@H](O)CO</smiles>

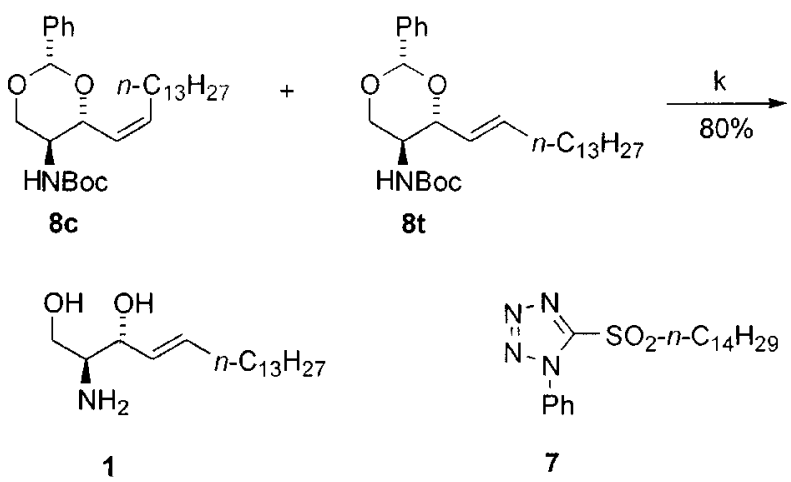

Scheme 1. (a) TIPSOIT, Et $\mathrm{N}_{3}, \mathrm{CI}_{2} \mathrm{Cl} \mathrm{l}_{2},-78$ to -20 " $\mathrm{C}$ : (b) $\mathrm{Cl}_{3} \mathrm{CCN}$, $\mathrm{NaH}, \mathrm{THF},-30^{\circ} \mathrm{C}$, then $n-\mathrm{Bu}{ }_{1} \mathrm{NF}_{3}-30^{\circ} \mathrm{C}$ : (C) IBr: $\mathrm{K}_{2} \mathrm{CO}, \mathrm{FtCN}$. $-78^{\circ} \mathrm{C}$ : (d) $\left(\mathrm{CF}_{3} \mathrm{CO}\right)_{2} \mathrm{O}, \mathrm{Ft}_{3} \mathrm{~N}, \mathrm{CH}_{2} \mathrm{Cl}_{2},-20^{\circ} \mathrm{C}$, then $\mathrm{NaT}$, DMF, 0

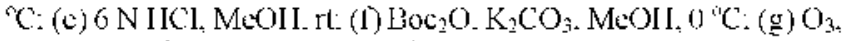
MoOII, $-78^{\circ} \mathrm{C}$, then $\mathrm{NaBH}_{4}$ o "C: (h) $p^{-\mathrm{T}} \mathrm{COH}$, PhCIIO. $\mathrm{CH}_{2} \mathrm{Cl}_{2}$, rt: (i) Swen oxid: (i) 7, KHMTS, DMF, $-60^{\circ} \mathrm{C}$, then aldelyde, $-600^{\circ} \mathrm{C}:(\mathrm{k}) \mathrm{CF}_{3} \mathrm{COOH}, \mathrm{H}_{2} \mathrm{O}, \mathrm{rt}$. 
<smiles>CC(C)(O)/C=C\[C@H]1O[C@@H](c2ccccc2)OC[C@H]1O</smiles><smiles>C=CC=C</smiles>

9

11

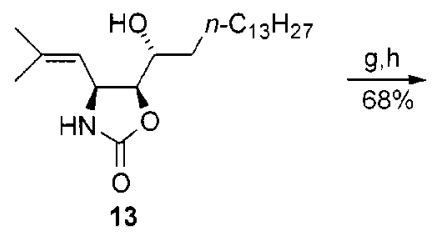<smiles></smiles><smiles>CC(C)(O)C(I)[C@H]1N=C(C(F)(F)Cl)O[C@@H]2CO[C@H](P)[C@@H]21</smiles>

10<smiles>CC(C)=CC1NC(=O)OC1[C@H]1CO1</smiles>

12<smiles>[14CH3]C[C@H](O)[C@H](O)[C@@H](N)CO</smiles>

2
Scheme 2. (a) $\left(\mathrm{Cl}_{3} \mathrm{CO}_{2} \mathrm{O}\right.$, Lla $\mathrm{N}_{3}, \mathrm{CH}_{2} \mathrm{Cl}_{2},-20{ }^{\circ} \mathrm{C}$, then Nal, DMl: 0 "C: (b) $6 \mathrm{~N} \mathrm{HCl}, \mathrm{MeOH}$, rt: (c) $\mathrm{Cb}_{7} \mathrm{Cl}, \mathrm{K}_{2} \mathrm{CO}, \mathrm{MeOH}_{3} 0^{\circ} \mathrm{C}:$ (d) 2,4,6- $\left.\mathrm{Me}_{3} \mathrm{C}_{6} \mathrm{H}_{2} \mathrm{SO}\right)_{2} \mathrm{Cl}$, DMAP, $\mathrm{Ft}_{3} \mathrm{~N}_{2} \mathrm{CH}_{2} \mathrm{Cl}_{2}, 0$ "C to tt: (e) $\mathrm{NaH}_{2}$

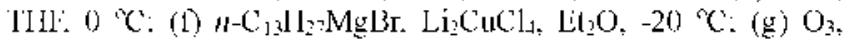

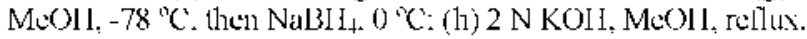

sphingosine 1 (mp. $78-80^{\circ} \mathrm{C}:\lfloor\alpha]_{13}^{25}-2.7 . \mathrm{c} 1,0 . \mathrm{CHCl}_{3}$ ) in $80 \%$ yicld. the spectroscopic and physical data of which are identical with those previously reported.

To synthesize D-ribo-phyiosphingosine 2. dihydro-l.3oxazinc 10. $\left([\alpha]_{12}^{23}-34,8, c \quad 1,0 . \mathrm{CHCl}_{3}\right)$ which was sccurcd in 2 steps and $89 \%$ yicld from diol 9 . was reductively eliminated. cxhaustively hydrolyzed. and the resulting amine was protected to render carbamate 11 (mp. 85-87 $\mathrm{C}:\lfloor\alpha\rfloor_{13}^{2 ?}$ $-32.1 . c 1.1 . \mathrm{CHCl}_{3}$ ) in $75 \%$ overall vicld (Schemc 2). Regioselective sulfonation of 11 followed by cyclization gave cpoxy oxazolidinone 12 (mp. $77-79^{\circ} \mathrm{C}:[\alpha]_{1)}^{+5}-13.8, \mathrm{c}$ 1.4. $\mathrm{CHCl}_{3}$ ) in $56 \%$ yield. The epoxy group of 12 was opened with tridecylmagnesium bromide in the presence of lithium tetracluorocuprate ${ }^{\text {ii }}$ to afford oxazolidimone 13 (mp. 57-59: $\left.\lfloor\alpha]_{13}^{2+}-8.2<0.7 . \mathrm{MeOH}\right)$ in $90 \%$ yield. Sequential subjection of 13 to ozonoly sis. $\mathrm{NaBH}_{\neg}$ reduction and basic hydrolysis produced D-ribo-phytosphingosine 2 (mp. 95-97 ${ }^{12} \mathrm{C}:\lfloor\alpha]_{12}^{2-1}+8.6 . c 0.7$. pyridine) in $68 \%$ yield the spectroscopic and physical data of which are in agreement with those reported in literatures. ${ }^{1 / \mathrm{h}, \mathrm{z}}$

Acknowledgment. This work was supported by CMDS and the Brain Korea 21 Project.

\section{References}

1. (a) Category: K.: Tori. K.: Kimura. Y.: Yoshida. T.: Nagasaki. T.: Minalo, J. J . Wed (hem. 1967, I0, 1147. (b) Grollmatr, A. P. .J. Biol. (hem. 1967, 242,3226, (c) Une\%awa, H.: Aovagi, T: Morishima, 11: Matswaki. M.: IJamada. H.: Takeuchi. T. $J$. Antibiot 1970. 23. 259. (d) Molyneux. R. J.: Tames. L. F. Science 1982. 216. 190. (e) Gruters. R. A.: Neefjes. I. J.: Tersmette. M.: de Gocde. R. E. Y.: Tulp. A.: Hulsman. H. G.: Micdema. F.: Plocgh.
H. L. . Hatwe 1987. 330. 74. (1) Omura. S.: Fujimoto. T.: Otogurro. K: Matsurahi, K: Moriguchi. R.: Tanaki. II: Sasaki. Y. J. Antibiot Chem 1991. H. 113. (g) Ilughes, A. B.: Rudge A. J. Nat. Prod. Rop. 1994. 11. 135.

2. (a) Kang. S. H.: Kim. G. T. Tktrdhedon Lutt, 1995. 36. 5049. (b) Kang. S. H.: Jun1. H.-S.: Youn. I.-H. Swhleft 1998. 1045 . (c) Kang. S. H.: Choi. H.-w: Kim. J. S.: Yout1. J.-H. Cheth. Conmum, 2000. 227.

3. (a) Kang. S. H.: Kyu I). II (Then. Commun 1996, 355 . (b) Kang. S. H.: J.ee. S. 13. Chom Commm 1998. 76l. (c) Kang S. II: Ilwang. Y. S.: Yourn. J.-I I. Tetrahedron Leff. 2001, +2. 7599).

4. Hannun. Y. A.: Bell. R. M. Scince 1989. 243. 500.

5. (a) Merrill. A. H.: Nimkar. S.: Menaldino. D.: Hannun. Y. A.: Loomis. C.: Bell. R. M.: Tyagi. S. R.: Lambeth. I. D.: Sterens. V. I.: I Hunter. R.: I iolla, 1). C Biochethisty 1989. 28. 31.38 . (b)

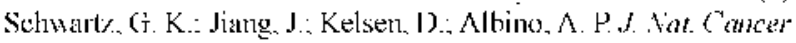
Inst. 1993, 85, 402

6. (a) Karlsson. K. A. In Biologrical Membranes: Chapman. D.. Ed.: Academic Press: London. 1982: Vol. 4. p 1. (b) Hakomori. S. In Handhook of lipid Researd, Iol. 3: Sphingolipid Biochemistr". Kanter . J. M.. I Iakomori. S.. Kds.: Plenum Press: Nen York, 1983. $\mathrm{pl}$.

7. (a) I lakomori, S. Am. Ro Biochem. 1981. 50,733. (b) Springer, T. A.: Lasky. L. A. Katme 1991. 349. 196. (c) Oliveira. A.: Spicgel. S. Glycoconjugate J. 1992. 9. 110 . (d) Merrill. A. H.. Ir:: Swecley: C. C. In Biochenisty of lipids, lipoproteins and Henbromes Vance. 1). Ii.. Vance. I. F... Eds.: Iilsevier Science: Amslerdam. 1996;, 309 .

8. Naroti. 1:- Morita, M: Akimolo. K: Koezuka, Y. Tetrolkedron 1994. 50.2771.

9. (a) Grag. H. S.: Sharma. M.: Bhakuni. D. S.: Pramanik. B. N.: Bose. A. K. Tetwhedron lett. 1992. 33. 1641. (b) Wong. C.-H.: Kamilakahara, II.: Suzuki. I: Nichigori. N.: Suruki. Y.: Kamie. 0. Angew (Them. fint Id fingl 1998, 37, 1524.

10. Kobavashi. S. I'uruta. T: I lavashi. 'T.: Nichijima. M.: I Ianada. K. J. Am. (hem. Soc. 1998. 120.908.

11. Marinicr. A.: Martel. A.: Banville. J.: Bachand. C.: Remillard. R.: Lapointe. P.: Turmel. B.: Menard. M.: Harte Ir.. W. E.: Kim Wright. I. I.: Todderud. (i.: Tramposch. K. M.: B3ajoralh. I.: Ilollenbaugh. J): Arullo, A. J A Hed (hem 1947, 40,3234

12. Costantino. V: Fattorusso, I.: Mangoni, A.: Rosa. M. I).: Ianaro. A.J.Am Chm Soc 1997. 119. 12465.

13. Natori. T:: Morita. M.: Akimoto. K: Koczuka. Y. Tetrolkedrom 1994.50 .2771 .

14. Iliguchi, R.: Inukai, K.: Jhou, I. X.: I Ionda, M. Komori. T.: Tsuji. S.: Nagai, Y. fiehigs lm. (hem. 1993. $35 \%$.

15. I.i. Il.: Maltsunaga. S.: Fusetani. N. Tetrahedron 1995, 51. 227.

16. (a) Koskinen. P. M.: Koskinen. A. M. P. Symthesis 1998. 1075. (b) Murakani. T.: Taguchi. K. Tetrulkedrom 1999. 55. 989. Clnung. S.$\mathrm{K}:$ Lee. J.M. Fetwahedron: . 1swmetry 1999. 10. 1441. (d) I lertweek. C. Boland. W. J. Org. Chem 1999, 6t. 426 . (e) Khiar. $\mathrm{N}$ : Singh. $\mathrm{K}$ : Garcia. M.: Martin-T omas, M. Fetrohedron $L_{t}$ 'ft. 1999, 40,577\%. (1) Nakamura. T., Shioyaki. M. Tatrahedron Leth. 1999. t0. 9063. (g) Shirota. O.: Nakanisti. K.: Bcrova. N. Tetratkedron 1999. 55. 13643. (h) Johnson. D. V: Fcltcri. U.: Griengl. H. Tenthedron 2(MMI. 56. 781. (i) Corey. E. I.: Choi. S. Fetrahedon lett. 20MO. H, 2765. (j) Azuma, it.: Tamagaki. S.:

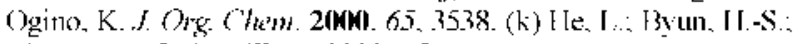
13ittmant, R. J. Ore Chem 2000. 65.7618.

17. Mancuso. A. T.: Swern. D. Symhesis 1981. 165.

18. Blackmore. P. R.: Colc. W. J.: Kocienski. P. I.: Morley. A. Sunlet 1998. 26.

19. (a) Dondoni, A.: Perrone, 1): Turturici. F.. . Chem. Soc., Pewin Trans. / 1997, 2389. (b) Spantu, P. Rassu, G.: Pinna, I .: I3altistini. I..: Cariraghi, G. Tetrahedon: Asymmetry 1997, 19, 32.37.

20. Tamura. M.: Kochi. I. Symbesis 1971. 305.

21. Li. Y.-L.: Mao. X.-H.: Wu. Y.-L. J. Chom. Soc. Ponh Trans. I 1995.1559. 